\title{
Federaciones y asociaciones deportivas en México: justificación y ordenamiento jurídico
}

Sports federations and associations in Mexico: justification and legal order

\author{
Daniel Ramiro Coral Muñoz ${ }^{1}$; Ricardo Navarro González ${ }^{1}$ \\ ${ }^{1}$ Facultad de Derecho Universidad Autónoma de Yucatán (UADY)
}

Detalles del artículo:

Número de palabras: 4.445; Tablas: 2; Figuras: 0; Referencias: 19

Recibido: julio; Aceptado: agosto; Publicado: diciembre

Conflicto de interés: El autor declara que no existen conflictos de interés.

Correspondencia del autor: Daniel Ramiro Coral Muñoz, danielcoralm@ hotmail.com

\section{Resumen}

Introducción: La práctica deportiva en México es realizada en abundancia a distintos niveles, desde el popular hasta el alto rendimiento o profesional. La naturaleza misma del deporte tiende a asociarse con la práctica adecuada, y al ser un derecho reconocido en la Constitución Mexicana necesita una figura jurídica que regule las actividades de cada deporte acorde a los lineamientos internacionales. En virtud de esta necesidad nacen las Asociaciones Deportivas Nacionales. Las Asociaciones Deportivas Nacionales, también conocidas como Federación, son la principal autoridad técnica de cada deporte en México, contando con una naturaleza jurídica dual. Es decir, de origen privado o civil pero que realizan actividades y reciben recursos de carácter público, debiendo ser revisados bajo un escrutinio jurisdiccional. Objetivo: El presente artículo tiene como objetivo, analizar la naturaleza jurídica y el origen constitucional de las asociaciones Deportivas Nacionales o Federaciones Deportivas. Mismas que, de acuerdo a la Ley General de Cultura Física y Deporte, cuentan con una función pública y privada dentro del desarrollo del deporte nacional. Así mismo, se estudiará de manera breve y concisa la definición del Sistema Nacional del Deporte (SINADE). Metodología: Se realiza un estudio descriptivo de esta figura jurídica, señalando su justificación constitucional, para después continuar con las generalidades, contexto y relevancia dentro del funcionamiento del deporte mexicano. Resultados: Las asociaciones deportivas nacionales son la máxima autoridad técnica de cada deporte, de conformidad con la Ley General de Cultura Física y Deporte, y de igual manera forman parte del sector privado. No obstante, coadyuvan con el Estado en el fomento del adecuado ejercicio del derecho al deporte y de otros valores vinculados al mismo, por lo que dicha actuación se considera de utilidad pública. Conclusiones: El actuar de las asociaciones deportivas nacionales debe ser siempre el correcto, porque a pesar de ser de naturaleza privada, son agentes colaboradoras del Estado y de esta manera, y de acuerdo con la Jurisprudencia, son autoridad responsable para efectos administrativos y del juicio de amparo.

Palabras claves: Derecho, Constitución, Federaciones deportivas, Derecho Deportivo. 


\section{Abstract}

Introduction: The practice of sports in Mexico is carried out in abundance at different levels, from popular to high performance or professional. The very nature of sports tends to be associated with proper practice, and since it is a right recognized in the Mexican Constitution, it needs a legal figure to regulate the activities of each sport according to international guidelines. By virtue of this need, the National Sports Associations were born. The National Sports Associations, also known as Federation, are the main technical authority of each sport in Mexico, with a dual legal nature. That is to say, of private or civil origin but which carry out activities and receive resources of a public nature, having to be reviewed under jurisdictional scrutiny. Objective: The purpose of this article is to analyze the legal nature and constitutional origin of National Sports Associations or Sports Federations. According to the General Law of Physical Culture and Sports, they have a public and private function within the development of national sports. Likewise, the definition of the National Sports System (SINADE) will be studied briefly and concisely. Methodology: A descriptive study of this legal figure is carried out, pointing out its constitutional justification, to then continue with the generalities, context and relevance within the functioning of Mexican sports. Results: The national sports associations are the highest technical authority of each sport, in accordance with the General Law of Physical Culture and Sports, and they are also part of the private sector. However, they cooperate with the State in the promotion of the adequate exercise of the right to sport and other values linked to it, so that such action is considered of public utility. Conclusions: The actions of the national sports associations must always be correct, because despite being of a private nature, they are collaborating agents of the State and in this way, and according to the Jurisprudence, they are a responsible authority for administrative purposes and in the amparo trial.

Key words: Law, Constitution, Sports Federations, Sports Law.

\section{INTRODUCCIÓN}

Existe una estrecha relación entre el Derecho y el Deporte, misma que de manera progresiva ha permitido consolidar el derecho del deporte. Respecto a esto, Gamero (2012) señala que el derecho del deporte es aquel que hace referencia al régimen jurídico aplicable a la actividad deportiva con especial consideración a competencias oficiales y de organización pública y privada.

Derivado de esto, en México existen diversos ordenamientos que pretenden regular la práctica deportiva. Siendo la principal de estas, la Ley General de Cultura Física y Deporte (LGCFD), misma que fue publicada en el Diario Oficial de la Federación el siete de junio del 2013.

Esta ley dentro del artículo 5 fracción V, define el deporte como:

"Deporte: Actividad física, organizada y reglamentada, que tiene por finalidad preservar y mejorar la salud física y mental, el desarrollo social, ético e intelectual, con el logro de resultados en competiciones". 
Definición que toma como base distintas acepciones como la de Parlebas (1993), quien define el deporte como una actividad física, competitiva, reglada e institucionalizada. Por su parte Hernández (1994), amplia la definición de Parlebas, añadiéndole a esta última que el deporte es una situación motriz de competición, reglada, de carácter lúdico e institucionalizada.

A pesar de que en la LGCFD se define y se regula el deporte, este nació al margen del Estado, pues su origen es asociativo privado. Fueron los individuos quienes inventaron de manera voluntaria las reglas de distintas disciplinas deportivas, es decir, ellos mismos decidieron asociarse en clubes, posteriormente en federaciones, para finalmente someterse de manera voluntaria al Comité Olímpico Internacional (Pérez, 2019).

Es así que del párrafo de Pérez se observa la tendencia por parte de los individuos de asociarse en agrupaciones para practicar el deporte en ciertos términos. Planteamiento que coincide con Muñiz (2015), que señala que la práctica deportiva implica una actividad social e institucionalizada, en donde los deportistas que pretenden llegar a competir en alguna disciplina, deben de afiliarse o asociarse en Instituciones que se encuentran reconocidas, debido a que estas organizan y promueven su respectiva disciplina.

Antes de analizar las cuestiones específicas de las Asociaciones Deportivas Nacionales, se debe realizar un breve análisis sobre el Sistema Nacional de Cultura Física y Deporte (SINADE) ya que resulta ser la base de la estructura deportiva de México.

\section{SISTEMA NACIONAL DE CULTURA FISICA Y DEPORTE (SINADE)}

Dentro del artículo número 10 de la LGCFD se puede observar la existencia de un Sistema Nacional del Deporte, mismo que otorga cabida a las Asociaciones Deportivas Nacionales dentro de la estructura del desarrollo deportivo en México. De acuerdo con lo anterior, el mencionado numeral establece que para la eficaz y eficiente promoción, fomento y estímulo de la cultura física y de la práctica del deporte en todas sus manifestaciones existirá un SINADE, que tendrá como objeto asesorar en la elaboración del Programa Nacional de Cultura Física y Deporte.

De esta manera, el SINADE resulta ser un órgano colegiado que se integra por las Dependencias, Organismos e Instituciones públicas y privadas, así como por las Sociedades, Asociaciones Nacionales y Consejos Nacionales del Deporte Estudiantil que están reconocidos por la LGCFD. A su vez, estas instituciones tienen como objetivo generar las acciones, financiamientos y programas necesarios para el fomento, ejecución, promoción, difusión y desarrollo de la activación física, la cultura física y el deporte de México (Muñiz, 2015).

En este sentido conforme al artículo 11 de la LGCFD, de manera gráfica se determina que son integrantes del SINADE los siguientes entes: 
Tabla 1. Integrantes del SINADE

La Comisión Nacional de Cultura Física y Deporte: es el organismo público que se encarga de conducir la política nacional en el ámbito de la cultura física y deporte.

Los órganos estatales, del Distrito Federal y Municipales de Cultura Física Deporte: son aquellos órganos de las entidades federativas y los municipios, que actúan en coordinación y colaboración con la CONADE.

El Comité Olímpico Mexicano: es una organización privada que representa al Comité Olímpico Internacional en nuestro país, y que se encarga de fomentar y velar por el desarrollo del deporte y el movimiento olímpico, así como la difusión de los ideales olímpicos.

El Comité Paralímpico Mexicano: es una organización privada que rige y organiza los deportes y modalidades deportivas para las personas con discapacidad en nuestro país.

Las Asociaciones Deportivas Nacionales: también conocidas como Federaciones, constituyen la máxima instancia técnica de la disciplina deportiva de que se trate, debiendo representar a un solo deporte en todas sus modalidades y especialidades.

Los Consejos Nacionales del Deporte de la Educación: se conforman por instituciones educativas de carácter público y privado de educación básica, media o superior, cuyo objeto es coordinar los programas emanados por la CONADE entre la comunidad estudiantil en sus respectivos niveles.

Para poder estudiar de manera clara y precisa a las Asociaciones Deportivas Nacionales, primero se deben establecer dos elementos jurídicos que resultan relevantes en México, tales como:

a) Derecho a la cultura física y deporte.

b) Derecho de Asociación.

\section{JUSTIFICACIÓN CONSTITUCIONAL DE LA CULTURA FISICA Y DEPORTE EN MÉXICO}

La Constitución Política de los Estados Unidos Mexicanos (CPEUM) de 1917 tiene 136 artículos a lo largo de su extensión, sin contar los artículos transitorios. Entre las 136 disposiciones normativas, se encuentra el artículo $4^{\circ}$, que desde el diez de diciembre del 2011 reconoce en su último párrafo el derecho a la actividad física y al deporte, situando a México entre los demás países latinoamericanos en otorgar rango constitucional a la actividad física y el deporte (Pachot, 2016). Esta aparentemente sencilla admisión resulta en diversas implicaciones de gran trascendencia, que serán desarrolladas a lo largo de este escrito.

En un primer momento, en virtud que el derecho a la actividad física y al deporte es un derecho previsto en la Constitución Federal Mexicana, debe esclarecerse primero que una constitución es: "un complejo normativo de naturaleza suprema y fundamental. Es un conjunto de normas dispuestas sistemáticamente con el propósito de organizar un estado; ellas regulan el uso del poder, garantizan el respeto de las libertades y permiten el ejercicio de derechos; son de jerarquía superior, permanentes, escritas, generales y reformables" (Arteaga, 2014 p.2).

Manteniéndolo sencillo, las constituciones democráticas modernas son las normas supremas y presentan dos clases o categorías de norma: la primera categoría constituye aquellas que organizan el poder legislativo, el poder ejecutivo y la administración de justicia, el Estado; lo principal de esto es la legitimación que la constitución le otorga a los demás 
poderes (Alexy 2002). La segunda categoría son aquellas normas que limitan o encausan el poder estatal, los derechos fundamentales.

Entonces, de las definiciones anteriores se concluye que toda vez que el derecho a la actividad física y al deporte, ordena:

"Toda persona tiene derecho a la cultura física y a la práctica del deporte. Corresponde al Estado su promoción, fomento y estímulo conforme a las leyes en la materia".

Se advierte que el Estado tiene la obligación de respetar el derecho a la cultura física y al deporte. Es decir, que ninguna autoridad puede realizar actos que impidan en su totalidad el derecho en comento, y de hacerlo debe estimarse inconstitucional, por contravenir el principio de supremacía constitucional. Al impedir el goce del derecho reconocido en la norma del último párrafo del artículo 4 de la Constitución.

\section{CONCEPTOS Y GENERALIDADES DE ASOCIACIONES Y FEDERACIONES DEPORTIVAS}

Antes de establecer conceptos y generalidades de las Asociaciones Deportivas Nacionales se advierte dentro de la CPEUM en su artículo noveno se prevé el derecho a asociarse, toda vez que dicho numeral a literalidad dispone:

"Artículo Noveno: No se podrá coartar el derecho de asociarse o reunirse pacíficamente con cualquier objeto lícito;",

Mientras que la doctrina, señala que el derecho de asociación consiste en la libertad de todos los habitantes para conformar, por sí mismos o por otras personas, entidades que teng an una personalidad jurídica distinta a la de sus integrantes, con el objeto y finalidad que sus integrantes libremente determinar, siempre que sea licito (Carbonell 2012).

En este sentido, el asociarse resulta ser un derecho para todos los mexicanos. De igual manera, una de las principales características del deporte es precisamente la existencia del asociacionismo deportivo. Pues dentro de los derechos del deportista se encuentra el de asociarse para la práctica del deporte y, en su caso, para la defensa de sus derechos. Refiriéndose a la posibilidad de asociarse con más personas para exigir el respeto de sus derechos como deportista en lo individual o a través de las asociaciones para formar libremente organismos deportivos (Muñiz, 2020).

Una Asociación Deportiva Nacional es una organización que tiene como objeto la regulación y organización de su deporte, está integrada por las asociaciones estatales, regionales o filiales de la misma disciplina deportiva, así mismo, expide normas y vigila la observancia del reglamento de su especialidad, pudiendo imponer medidas disciplinarias por infracciones a su normatividad (Fuertes, 1992). 
Las federaciones deportivas son el eje del deporte competitivo en la práctica totalidad de los países. A pesar de que habitualmente se trata de organismos con funciones públicas delegadas y sin ánimo de lucro, su gestión debería basarse en principios similares a los de empresas de cualquier otro sector económico, tales como: excelencia, eficiencia y buen gobierno (Vega, 2019).

En cuanto al concepto de una asociación deportiva, la LGCFD en su numeral 43 establece que las asociaciones deportivas registradas por la CONADE serán las personas morales, sin importar su estructura, naturaleza y denominación jurídica, que conforme a su objeto social promuevan, difundan, practiquen o contribuyan a desarrollo del deporte, sin fines preponderadamente económicos (Hernández, 2018).

Ahora bien, el artículo 46 de la LGCFD clasifica las asociaciones deportivas en:

Tabla 2. Clasificación Asociaciones Deportivas

\begin{tabular}{l} 
Equipos o clubes deportivos \\
Ligas deportivas \\
Asociaciones Deportivas, Municipales, Estatales o Regionales, Consejos nacionales del deporte \\
Estudiantil \\
Asociaciones Deportivas Nacionales - Organismos Afines \\
\hline
\end{tabular}

En este sentido, las Asociaciones Deportivas Nacionales resultan ser la máxima instancia técnica de la disciplina deportiva, debiendo representar a un solo deporte en todas sus modalidades y especialidades.

\section{DISCUSIÓN}

De esta manera, se ha aceptado que en virtud de que las asociaciones del sistema nacional deportivo forman parte de políticas públicas para el desarrollo de reglas o técnicas para el sustento de un deporte; no pueden desconocer los principios constitucionales, ni vulnerar los derechos fundamentales de sus destinatarios, cuando establezcan sus reglamentos. En caso de que el reglamento implique discriminación u opacidad que impida el goce del derecho humano a la cultura física y a la práctica del deporte de una persona, entonces independientemente de que se trate de una relación entre particulares (SCJN 2020), lo que haría procedente el juicio de amparo para la revisión y en su caso impugnación de estos.

De acuerdo con Pérez (2019), las federaciones deportivas han aspirado desde sus inicios a gobernar de manera autónoma la práctica deportiva, por lo que se materializa la relevancia de estas instituciones. Toda vez que las asociaciones deportivas nacionales cuentan con reconocimiento como autoridades para efectos del juicio de amparo cuando sancionan cuestiones disciplinarias.

Otra cuestión relevante, es que dentro del artículo 52 de la actual LGCFD se reconoce a las asociaciones deportivas nacionales como la máxima instancia técnica de su disciplina y establece que deberán representar a un solo deporte en todas sus modalidades y especialidades, en los términos del reconocimiento de su respectiva federación deportiva internacional. Además, son las únicas facultadas para convocar a competencias realizadas bajo la 
denominación de "Campeonato Nacional" con estricto apego a los estatutos y reglamentos aplicables, y de acuerdo con los criterios que fije la CONADE.

Un ejemplo evidente y hasta hiperbólico, es que sería perjudicial y sin fundamento que un titular de asociación o federación deportiva emitiera una convocatoria que en esencia perjudique, vulnere, excluya o discrimine a uno o más individuos para realizar competencias deportivas, o que algún ente administrativo ordene obstáculos arbitrarios para el ejercicio de este derecho. El ejemplo es exagerado, pero ilustra la relación entre la subordinación de las leyes secundarias y los actos de demás autoridades.

Concatenado con esto, se destaca que los Tribunales Colegiados de Circuito han fallado en concesión de que también los particulares en esferas relacionadas al deporte pueden violar derechos humanos, por lo que sus actos pueden ser sometidos al escrutinio constitucional, incluso al juicio de amparo.

La razón de esto es que, al ser la máxima instancia de su respectiva disciplina, independientemente de que su constitución pueda ser de carácter civil, los actos u omisiones son unilaterales y obligatorios y trastocan la situación jurídica de las personas. Por lo que, si una asociación deportiva sin justificación válida sancionase a una persona de tal forma que no pudiera competir, entonces este acto es sujeto de impugnación por el juicio de derechos humanos en México, conocido también como juicio de amparo.

Entendiendo al juicio de amparo mexicano, parta todos los efectos de este trabajo "como el medio de control de constitucionalidad, convencionalidad y legalidad de los actos $\mathrm{u}$ omisiones provenientes de autoridades o particulares, previsto en favor de las personas (físicas o morales), cuyo objetivo es proteger los derechos humanos y las garantías otorgadas para su protección por la Constitución y los Tratados Internacionales de los que México sea parte, restituyéndolos, en caso de que se procedente, de manera integral el goce del derecho violentado" (Coaña, 2019 p.40).

De esta manera, se advierte la relevancia de las posturas del poder judicial de la federación, en razón de que ha confirmado a través de diversas jurisprudencias la naturaleza jurídica dual de las asociaciones/federaciones deportivas nacionales, mismas que presentan diversos argumentos importantes como el mencionado en el párrafo anterior, misma que se transcribe:

\section{"Asociaciones deportivas nacionales. Realizan actos equivalentes a los de autoridad para efectos del juicio de amparo cuando ejercen la potestad disciplinaria, porque son la máxima instancia técnica de su disciplina y representan a un solo deporte en todas sus modalidades y especialidades"}

Las funciones públicas de carácter administrativo delegadas a dichas asociaciones cuando actúan como agentes colaboradores del Gobierno Federal (que se considera de utilidad pública), están determinadas en una norma general que les confiere atribuciones para actuar como autoridades del Estado, cuyo ejercicio tiene un margen de discrecionalidad, en tanto pueden decidir si las ejercen o no y en qué sentido. Además, las asociaciones deportivas nacionales son la máxima instancia técnica de su disciplina 
y representan a un solo deporte en todas sus modalidades y especialidades, de modo que sus decisiones están revestidas de un grado de imperatividad; por tanto, pueden dictar, ordenar, ejecutar o tratar de ejecutar algún acto en forma unilateral y obligatoria, o bien, omitir actuar en determinado sentido, lo que se traduce en que, con independencia de que formalmente puedan estar constituidas como una asociación de carácter civil, pueden realizar actos equivalentes a los de autoridad en los que afecten derechos creando, modificando o extinguiendo situaciones jurídicas; de manera que cuando actúan así u omiten hacerlo, deben considerarse como particulares equiparados a una autoridad responsable para efectos del juicio de amparo.

\section{PLENO EN MATERIA ADMINISTRATIVA DEL PRIMER CIRCUITO}

Esta tesis se publicó el viernes 12 de agosto de 2016 a las 10:20 horas en el Semanario Judicial de la Federación y, por ende, se considera de aplicación obligatoria a partir del lunes 15 de agosto de 2016, para los efectos previstos en el punto séptimo del Acuerdo General Plenario 19/2013."

Como se ha mencionado las asociaciones deportivas nacionales, también conocidas como federaciones, tienen una naturaleza jurídica dual. Toda vez que son asociaciones civiles con personalidad jurídica propia y se rigen por sus propios estatutos sociales de acuerdo con el Código Civil Federal y la LGCFD. De igual manera, cuentan con reglamentos técnicos y reglamentos deportivos que establecen órganos propios, por lo que desarrollan su actividad deportiva, tomando decisiones relativas al deporte a su cargo (González, 2018).

De esta forma, la LGCFD señala que las federaciones debidamente reconocidas, además de sus propias atribuciones, ejercen por delegación funciones públicas de carácter administrativo. Actuando en este caso como agentes colaboradores del gobierno federal, por lo que dicha actuación se considerará de utilidad pública.

Dentro de las obligaciones de estas federaciones se tiene el garantizar el cumplimiento efectivo de las funciones que como colaboradoras de la administración pública federal les son delegadas a las federaciones deportivas nacionales. La CONADE, con absoluto y estricto respeto a los principios de auto organización, compatibles con la vigilancia y protección de los intereses públicos, podrá llevar a cabo acciones de fiscalización, supervisión, evaluación y vigilancia de los recursos públicos. Esto resulta obvio, pues al otorgar la CONADE recursos económicos federales, por obligación tiene que fiscalizar su buena y transparente aplicación.

Como prueba de esto, Pachot (2016) ha señalado la necesidad de someter al control jurisdiccional las acciones y omisiones, tanto de los órganos públicos, como de la esfera privada, sobre todo por los abusos que podían cometer las segundas y por su naturaleza de entes privados, escapaban de cualquier control judicial cuando vulneraban derechos humanos.

No obstante, el derecho constitucional del deporte y actividad física en México aún deja mucho que desear. Toda vez que los criterios de los Tribunales Colegiados de Circuito pueden ser considerados de vanguardia, lo cierto es que, al ser Tribunales Colegiados de Circuito, su obligatoriedad se encuentra constreñida al mismo circuito del que emanaron. Por lo que Tribunales Colegiados de otros circuitos, en su discrecionalidad, pueden estar de acuerdo y 
aplicar dichos criterios, pero también están facultados para discernir y resolver de forma menos protectora para las personas, en pro de las asociaciones deportivas.

Por lo tanto, sería oportuno que la Suprema Corte de Justicia de la Nación tenga la oportunidad de producir jurisprudencia que fije el precedente más garantista y como consecuencia; avanzar la esfera de protección que genera el último párrafo del artículo 4 constitucional, para que proteja a las personas de entes públicos y privados de cualquier acto injusto.

"Nunca te guíes por la ley del encaje, que suele tener mucha cabida con los ignorantes que presumen de agudos. Hallen en ti más compasión las lágrimas del pobre, pero no más justicia, que las informaciones del rico. Procura descubrir la verdad por entre las promesas y dádivas del rico, como por entre los sollozos e importunidades del pobre." (Cervantes, 1614).

\section{CONCLUSIONES}

- Debido a la característica de la asociación en el deporte, la simple actividad física no debe confundirse con el Deporte, debido a los elementos esenciales del mismo: Actividad física, reglada, organizada e institucionalizada o asociada.

- Las Asociaciones Deportivas Nacionales son personas morales, esto significa que necesitan obligatoriamente un contrato social, acta constitutiva, o documento similar, como presupuesto necesario para dotarlas de personalidad jurídica.

- Las asociaciones y federaciones deportivas pueden tener cualquier denominación, estructura o naturaleza jurídica, siempre y cuando tengan como objeto social el promover, difundir o desarrollar el deporte en cualquiera de sus vertientes.

- Las Asociaciones Deportivas Nacionales al ser la máxima instancia de su respectiva disciplina, cuentan con reconocimiento como autoridades para efectos del juicio de amparo, a pesar de constituirse de carácter civil o privado.

- Las Asociaciones Deportivas Nacionales forman parte del sector privado, no obstante, coadyuvan con el Estado en el fomento del adecuado ejercicio del derecho al deporte y de otros valores vinculados al mismo, por lo que dicha actuación se considerará de utilidad pública.

- Las Asociaciones Deportivas Nacionales no deben violar derechos humanos, por lo que sus actos pueden ser sometidos al escrutinio constitucional. 


\section{REFERENCIAS}

Alexy, R. (2002). Derechos Fundamentales, Ponderación y Racionalidad, México, Centro de Estudios Jurídicos Carbonell A.C.

Arteaga, E. (2014). Derecho Constitucional. México: Oxford.

Carbonell, M. (2012). Los derechos fundamentales en México, México, Porrúa. p. 480

Cervantes, M. (1614). Segunda Parte del Ingenioso Caballero Don Quijote de la Mancha. Estados Unidos de América: Vintage Español. pp. 833 y 834.

Coaña, L. (2019). El Juicio de Amparo. México: Tirant Lo Blanch. p. 40

Constitución Política de los Estados Unidos Mexicanos, Diario Oficial de la Federación, México, 5 Febrero 1917.

Fuertes López, M, (1992). Asociaciones y Sociedades Deportivas, España.

Gamero Casado, Eduardo. (2012). Fundamentos de derecho deportivo, Ed. Tecnos, España, , p. 59

González Guerrero, N. (2018). Régimen jurídico de la Comisión de Apelación y Arbitraje del Deporte de México y una propuesta de mejora. Universidad de Lleida, España.

Hernández González, D. (2014). Derecho del Deporte, México, Editorial Flores.

Hernández González, D. (2018). Asociaciones y sociedades Deportivas, México, Editorial Porrúa.

Hernández Moreno, J. (1994). Fundamentos del deporte. Análisis de las estructuras del juego deportivo, Barcelona, Editorial Inde. https://bit.ly/3qwQy8R

Ley General de Cultura Física y Deporte, Diario Oficial de la Federación, México, 7 de junio de 2013.

Muñiz Dominguez, P. (2015). Las asociaciones deportivas nacionales como agentes colaboradoras del gobierno federal, México, Revista Académica de la Facultad de Derecho de la Universidad La Salle, pp. 145-161 https://bit.ly/3wwyMUX

Muñiz Dominguez, P., (2020, 19 de junio). Derechos de los deportistas: ¿cuáles son en México?, Journey App, Recuperado de: https://bit.ly/3xChUxx

Pachot, K., (julio 2016). El Derecho Constitucional Al Deporte En La Doctrina y El Derecho Comparado. Cuestiones Constitucionales Revista Mexicana de Derecho Constitucional, Núm. 35, pp. 119-150. https://bit.ly/3mR9f4H

Parlebas, P. (1993). Problemas del juego en la Educación Física, Actas Primer Congreso Argentino de Educación Física y Ciencias. Departamento de Educación Física, FHCE-UNLP, La Plata. pp. 393-410. https://bit.ly/39SOrX7

Pérez Triviño, J. (2019). Resolución de conflictos en el deporte: análisis y propuestas. Madrid, Editorial Reus.

Vega, P; Pérez-González, B; Fernández-Luna, A; Burillo, P. (2019). Diversidad de género en las juntas directivas de federaciones deportivas: resultados económicos y operacionales, Apuntes. Educación Física y Deportes, n. ${ }^{\circ} 137,3$.er trimestre (julio-septiembre), pp.115-128 https://bit.ly/3xfbowp

\section{JURISPRUDENCIAS}

"ASOCIACIONES DEPORTIVAS NACIONALES. REALIZAN ACTOS EQUIVALENTES A LOS DE AUTORIDAD PARA EFECTOS DEL JUICIO DE AMPARO CUANDO EJERCEN LA POTESTAD DISCIPLINARIA, PORQUE SON LA MÁXIMA INSTANCIA TÉCNICA DE SU DISCIPLINA Y REPRESENTAN A UN SOLO DEPORTE EN TODAS SUS MODALIDADES Y ESPECIALIDADES”, Pleno de Circuito, Gaceta del Semanario Judicial de la Federación, Décima Época, Tesis: PC.I.A. J/79 A (10a.), Jurisprudencia, Libro 33, agosto de 2016, Tomo III, página 1382. https://bit.ly/3huTJLG

“DERECHO HUMANO A LA CULTURA FÍSICA Y A LA PRÁCTICA DEL DEPORTE. SI LA EXPEDICIÓN DE LA REGLAMENTACIÓN TÉCNICA DEPORTIVA IMPLICA DISCRIMINACIÓN U OPACIDAD QUE LO IMPIDA, COBRAN VIGENCIA LOS DERECHOS FUNDAMENTALES EN LAS RELACIONES ENTRE PARTICULARES EN SU EJERCICIO”, Tribunal Colegiado de Circuito, Gaceta del Semanario Judicial de la Federación, Décima Época, tTesis: XVII.2o.C.T.3 CS (10a.), Aislada, Libro 74, enero de 2020, Tomo III, página 2564. https://bit.ly/3yOXhOT 